\title{
Block the zinc, starve the virus
}

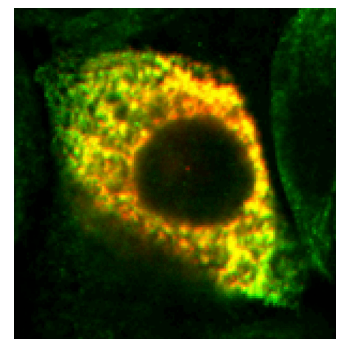

EVERs keep zinc out of the nucleus by trapping zinc transporters (green) at the ER (red).
Zinc might strengthen our immune systems and fortify our bones, but it also helps a cancer-causing skin virus take hold, according to Lazarczyk et al. (page 35).

Most people harbor within their skin a human papillomavirus (HPV) subtype called HPV EV. Although this virus is usually harmless, certain infected individuals develop wart-like skin lesions that eventually grow into tumors. These rare, susceptible individuals have mutated versions of ER membrane proteins known as EVER1 and EVER2.

Lazarczyk et al. now show that normal versions of EVER proteins counter the virus by depriving the cell's nucleus of zinc- a known transcription booster. Zinc is shuttled into the nucleus by a transporter called ZnT-1. But the team found that EVERs bound and retained $\mathrm{ZnT}-1$ at the ER, thus keeping the level of zinc in the nucleus low.

Cells containing mutated versions of EVERs had abnormally high levels of nuclear zinc, which activated proproliferation transcription factors, thus increasing the host cell's ability to seed tumors. Adding back functional EVERs reduced cellular proliferation. As the extra zinc also activated cellular transcription factors required for viral replication, EVERs might normally block viral replication as well.

Unlike HPV EV, the group found, other HPVs fought back against EVER proteins. One version that causes genital cancer, for instance, manufactured a protein that disrupted the EVER-ZnT-1 complex and freed zinc for nuclear entry. JEM

\section{New vaccines may provide full coverage}

On page 117, Giefing et al. report the discovery of two new vaccine candidates that might protect humans against all 90 versions of a deadly bacterium.

This bacterium, Pneumococcus, invades the lungs, blood, and eventually brain to cause pneumonia, sepsis, and meningitis, respectively. Surface proteins from this bug are currently being used as vaccines to induce the body to produce protective antibodies. But these vaccines, which comprise proteins from only a few types of Pneumococcus, are not effective against other variants, due to extreme sequence diversity in the surface proteins.

Giefing et al. now identify two bacterial antigens that are nearly identical among all pneumococcal strains and that induce potent antibodies in vivo. The authors hunted for conserved bacterial antigens that would be exposed on the pathogen during disease. Antibodies isolated from exposed but healthy humans and from those recovering from infection were used to identify pneumococcal proteins that were targeted by the protective antibody response.

Both of the newly identified antigens induced antibodies that protected immunized mice against several other pneumococcal variants. The antigens might be potent targets because they come from a protein that is essential for bacterial growth and survival and thus unlikely to mutate. The team is currently testing their vaccines in clinical trials. JEM

\section{An inflammation-enhancing virus}

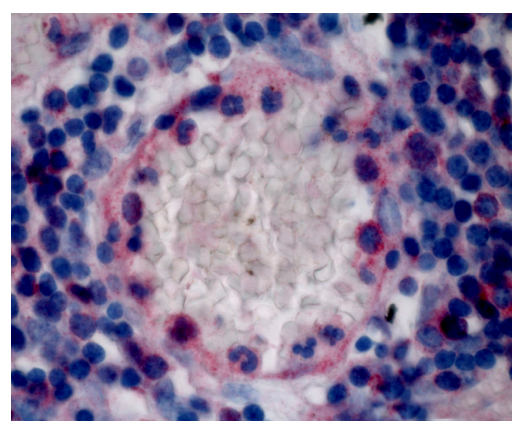

HCMV induces infected cells to produce leukotrienes (pink) that attract more inflammatory cells (dark blue).
A dormant virus that is awoken by inflammation enhances this potentially dangerous immune response to remain active, say Qiu et al. (page 19).

The human cytomegalovirus (HCMV) switches off its own replication after infection to stay off the immune system's radar. Unlike other dormant viruses that require a weakened immune system to reactivate, HCMV thrives amidst a roaring immune response. The virus replicates when its host cells-monocytes and other inflammation-causing cell typesproliferate. These cells produce cytokines such as TNF that directly stimulate the promoters of some HCMV genes.

Active HCMV infections are thus commonly found within the inflamed tissues of patients suffering from chronic inflammatory diseases, such as atherosclerosis. But whether the reactivated virus is just a lucky beneficiary of local inflammation or actively perpetuates inflammation was under debate.

Qui et al. now find that HCMV enhances inflammation by coercing nearby noninflammatory cells to join the fray. Smooth muscle cells isolated from inflamed tissues harbored active HCMV and produced leukotrienes-powerful proinflammatory lipids. Leukotriene production had been thought to be restricted to immune cells. How the virus reprograms the previously harmless muscle cells to become inflammatory is not clear.

The virus's escalation of inflammation probably amplifies its own growth and spread; smooth muscle cells, monocytes, and other HCMV host cells migrate when activated and might thereby seed new sites of viral activity. JEM 